\title{
Iron lines from transient and persisting hot spots on AGN accretion disks
}

\author{
R. W. Goosmann ${ }^{1,2}$, M. Mouchet ${ }^{2,3}$, B. Czerny ${ }^{2,4}$, M. Dovčiak ${ }^{1}$, V. Karas ${ }^{1}$, A. Różańska ${ }^{2,4}$, and A.-M. Dumont ${ }^{2}$ \\ 1 Astronomical Institute, Academy of Sciences, Boční II 1401, 14131 Prague, Czech Republic \\ email:goosmann@astro.cas.cz \\ 2 Observatoire de Paris, Section de Meudon, LUTH, 5 place Jules Janssen, 92195 Meudon Cedex, France \\ 3 Laboratoire Astroparticule et Cosmologie, Université Paris 7, 10 rue Alice Domon et Léonie Duquet, 75205 Paris Cedex 13, France \\ ${ }^{4}$ Copernicus Astronomical Center, Bartycka 18, 00716 Warsaw, Poland
}

Received 13 July 2007 / Accepted 30 August 2007

\begin{abstract}
Aims. We model the X-ray reprocessing from a strong co-rotating flare above an accretion disk in active galactic nuclei. By performing detailed radiative transfer computations we explore the horizontal structure and evolution of the underlying hot spot. The main goal is to study how the resulting spectral features manifest themselves in short exposure time spectra.

Methods. We analyze both the vertical and the horizontal spot structure and its dynamical reprocessed spectrum. To obtain the spectral evolution seen by a distant observer, we apply a general relativity ray-tracing technique. We concentrate on the energy band around the iron K-line, where the relativistic effects are most pronounced. Persistent flares lasting for a significant fraction of the orbital time scale and short, transient flares are considered.

Results. In our time-resolved analysis, the spectra recorded by a distant observer depend on the position of the flare/spot with respect to the central black hole. If the flare duration significantly exceeds the light travel time across the spot, then the spot horizontal stratification is unimportant. On the other hand, if the flare duration is comparable to the light travel time across the spot radius, the lightcurves exhibit a typical asymmetry in their time profiles. The sequence of dynamical spectra proceeds from more strongly to less strongly ionized re-emission. At all locations within the spot the spectral intensity increases towards edge-on emission angles, revealing the limb brightening effect.

Conclusions. Future X-ray observatories with significantly larger effective collecting areas will enable to spectroscopically map out the azimuthal irradiation structure of the accretion disk and to localize persistent flares. If the hot spot is not located too close to the marginally stable orbit of the black hole, it will be possible to probe the reflecting medium via the sub-structure of the iron K-line. Indications for transient flares will only be obtained from analyzing the observed lightcurves on the gravitational time scale of the accreting supermassive black hole.
\end{abstract}

Key words. radiative transfer - accretion, accretion disks - galaxies: active - galaxies: Seyfert - X-rays: galaxies

\section{Introduction}

The broad shape of the iron line complex seen in about $25 \%$ of all active galactic nuclei (AGN) is commonly assumed to be due to relativistic effects acting in the immediate vicinity of the central, supermassive black hole (e.g. Tanaka et al. 1995; Iwasawa et al. 1999; Wilms et al. 2001; Lubiński \& Zdziarski 2001; Fabian et al. 2002; Young et al. 2005; Guainazzi et al. 2006). The line is expected to be a reprocessing feature emitted by the surface of the irradiated inner accretion disk. An attractive scenario for the origin of the $\mathrm{X}$-ray irradiation envisions the reconnection of magnetic loops above the disk surface (see e.g. Galeev et al. 1979; Abramowicz et al. 1991; Haardt et al. 1994; Collin et al. 2003, Merloni \& Fabian 2001). The line emitting region lies at distances of only a few gravitational radii $R_{\mathrm{g}}=\frac{G M}{c^{2}}$ from the disk center.

Recently, some spectrally resolved variability of the line complex was observed for several objects (i.e. Iwasawa et al. 2004; Turner et al. 2006; Miller et al. 2006; Tombesi et al. 2007; Miniutti et al. 2007). For two of them, NGC 3516 and NGC 3783, the iron line feature appears to vary systematically in flux and centroid energy. The variations happen on characteristic time-scales of $25 \mathrm{ks}$ and $27 \mathrm{ks}$ respectively (Iwasawa et al. 2004;
Tombesi et al. 2007). Iwasawa et al. (2004) suggest a promising explanation for this type of line behavior invoking an X-ray flare, which is co-rotating with the accretion disk. The flare irradiates the disk surface creating a hot spot. In the case of NGC 3516 the flare and the underlying hot spot are located at (7-16) $R_{\mathrm{g}}$ from the black hole. The systematic changes of the reprocessed spectrum are then explained by the orbital motion of the disk and the resulting time-dependent relativistic modifications.

The above mentioned model requires a prescription for the reprocessed emission from the disk. The modeling of the reprocessed spectra is often carried out with considerable simplifications. Many computations assume either a uniform density of the disk atmosphere, or semi-isotropic illumination and "observation". The angular dependence of the reflected component formed by a constant density partially ionized medium was studied starting with Matt et al. (1993a,b), Życki et al. (1994), Życki \& Czerny (1994). Then, reprocessing from a medium in hydrostatic equilibrium was modeled by Raymond (1993), Nayakshin et al. (2000), Ballantyne et al. (2001), Ballantyne \& Ross (2002), Różańska et al. (2002). Nayakshin \& Kazanas (2002) pioneered the simplified time-dependent reflection studies for photoionized accretion disks. Goosmann et al. (2006a) studied rms spectral variability from a distribution of flares with vertically stratified 
spots underneath. None of those models considered a horizontal stratification of the illuminated spot as suggested by Goosmann et al. (2006b).

In this paper we add considerable sophistication to the flare model by studying in detail the Compton reflection/reprocessed component coming from a single orbiting spot. Since the spot properties have a significant gradient from the spot center to the border, we take both the vertical stratification of the reprocessing material and the horizontal stratification into account. The locally re-emitted spectrum is computed at various emission angles, and combined with a relativistic ray-tracing method (Dovčiak et al. 2004; Dovčiak 2004) to model the spectra seen by a distant observer.

We model the observed time evolution of the hot spot emission for two cases: a persisting flare lasting for a significant fraction of one orbital period at the given disk radius and short-term flares with durations of a few hundreds of seconds. The first case applies to recently reported observations of exceptionally powerful flares lasting for minutes to hours. Examples were given e.g. for MCG-6-30-15 (see Fig. 7 in Ponti et al. 2004) and NGC 5548 (see Fig. 1 in Kaastra et al. 2004). For the short-term flares, the horizontal stratification of the spot becomes important. During the onset and fading of the flare, the spot evolves from the center, where the first primary photons arrive, to the border. We calculate this effect presenting the resulting lightcurves and averaged spectra for short-term flares. We show that future data from the planned XEUS satellite will be of enough quality to be compared with our models.

The structure of the paper is as follows: Sect. 2 describes our model. The obtained locally emitted spectra are presented in Sect. 3, and those seen by a distant observer in Sect. 4. The importance of the studied effects is discussed in Sect. 5. Broader implications and some final conclusions are given in Sect. 6.

\section{The model}

Our method to compute the local spectra of the hot spot is similar to the one described in Collin et al. (2003). First, we compute the vertical density structure of the disk at a given radius $R$ assuming that the disk is in hydrostatic equilibrium without any external irradiation. The density structure of the surface layers are then used to conduct detailed radiative transfer computations. We take into account the intrinsic time evolution of a flare and we compute the spectral changes as seen by a distant observer. We apply a ray-tracing technique to include appropriate timedelays, general relativity effects, and the orbital motion of the hot spot.

In this paper, we assume for all computations the metric of a Schwarzschild black hole with $M=10^{8} M_{\odot}$ and an accretion rate $\dot{m}=0.001$ in units of the Eddington accretion rate. The surrounding disk with the viscosity parameter $\alpha=0.1$ is considered at the radii $7 R_{\mathrm{g}}$ and $18 R_{\mathrm{g}}$.

\subsection{The vertical disk structure}

Following the argument made by Collin et al. (2003) we assume that the flare is much shorter than the characteristic timescale for the restoration of the hydrostatic equilibrium. Hence, we assume that there is no external irradiation before the onset of the flare and the computation of the initial disk profiles is based only on the emission coming from the standard $\alpha$-disk at the given radius $R$. This condition is safely fulfilled if we assume that the flare duration is less than the orbital timescale at $R$.
We assume that the disk atmosphere is a plane-parallel, vertically stratified slab. The density, temperature, and radiation flux profiles are computed using an advanced version of the code described in Różańska \& Czerny (1996), Różańska et al. (1999), and Różańska et al. (2002). The code computes the hydrostatic balance of the medium in the gravitational field of the black hole, thereby including the relativistic corrections to the equations of the vertical disk structure (Novikov \& Thorne 1973; Page \& Thorne 1974). The radiation inside the medium is transported by diffusion using the Rossland approximation.

\subsection{Computing the local spectra}

Having obtained the vertical density profile of the disk, we compute the local reprocessed spectra expected after the onset of the flare. Since the principal spectral features are produced within a few Thomson optical depths $\tau_{\text {ES }}$ from the disk surface, we only consider the upper layers up to $\tau_{\mathrm{ES}}=7$ for the radiative transfer calculations. We call this upper layer the disk atmosphere and assume that it is heated from underneath by the thermal radiation of the underlying disk bulk. The temperature and incident flux $F_{\text {disk }}$ of the disk bulk are computed when obtaining the vertical disk structure. From above, the disk atmosphere is irradiated by the flare located at a given height $h$ that is measured from the disk surface where the flare causes the incident flux $F_{\text {inc }}$.

We assume that half of the primary radiation reaches the disk atmosphere. The main fraction of the flux is reprocessed directly underneath the flare source. The radiation emitted further sideways reaches the disk farther away from the flare and so it becomes geometrically diluted. Therefore, we can define a limited hot spot on the disk surface. The spot is centered on the orthogonal projection of the point-like flare source. The circumference of the spot and the flare source define an irradiation cone with half-opening angle $\theta_{0}$. We neglect any irradiation outside this cone, thus we only consider reprocessed radiation coming from inside the spot.

The distance $R$ of the flare from the central black hole is measured for the spot center. We limit the flare cone to $\theta_{0}=60^{\circ}$ and the height of the source to $h=0.5 R_{\mathrm{g}}$. We thus assume that the spot size is relatively small and neglect the change in the disk's internal properties across the spot. It remains to be seen whether such an assumption is justified. The MHD simulations of Miller \& Stone (2000) and Blaes et al. (2007) suggest magnetic loop heights that are by $\sim 2-3$ times higher than the disk thickness, while Turner (2004) found little evidence for magnetic loops rising so high.

The total incident flux, $F_{\text {inc }}^{\text {tot }}$, of the whole spot is parameterized by the ratio between the total incident flux and the internal flux emitted by the disk, $F_{\text {disk }}$. We set $F_{\text {inc }}^{\text {tot }} / F_{\text {disk }}=144$. This ratio ensures that the incident radiation by the flare is much stronger than the disk emission (the same value was used earlier by Ballantyne et al. 2001a; and Collin et al. 2003). The local incident flux, $F_{\text {inc }}$, decreases with the distance from the spot center and the incident angle, $\theta_{\mathrm{i}}$ increases as illustrated in Fig. 1. We divide the hot spot into five concentric rings defining equally spaced solid angles with respect to the flare source. Integrated over its surface each ring thus receives the same energy per unit time. We solve the radiative transfer for each ring individually using separate values of $F_{\text {inc }}$ and $\theta_{\mathrm{i}}$. Across a given ring we assume that $F_{\text {inc }}$ and $\theta_{\mathrm{i}}$ are constant.

The spectral shape of the primary radiation is modeled as a power law extending from $1 \mathrm{eV}$ to $100 \mathrm{keV}$ with the photon index $\Gamma=1.9$. The radiative transfer simulations are then conducted by the coupled use of the codes TITAN and NOAR 


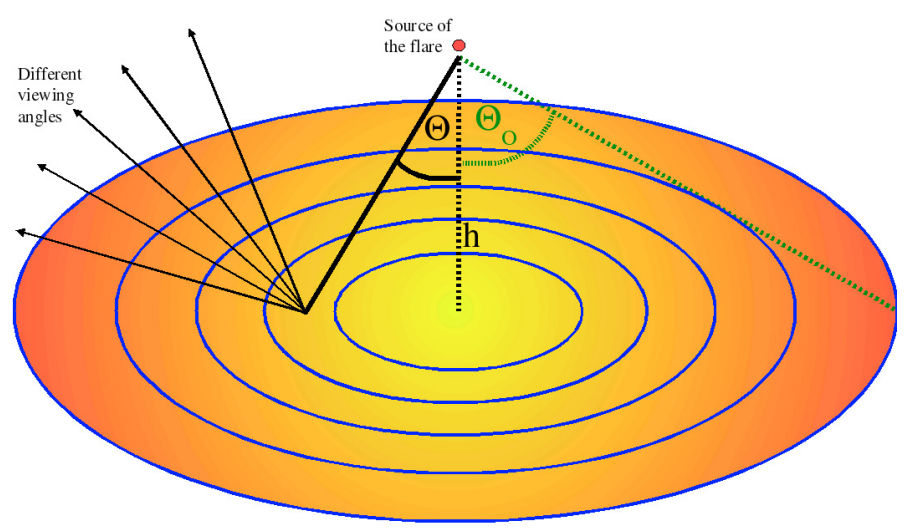

Fig. 1. Illustration of the hot spot at the disk surface underlying the flare. The illuminated region is divided into concentric rings of equally intercepted fractions of the flare luminosity.

described by Dumont et al. (2000) and updated in Dumont et al. (2003). The code TITAN calculates the vertical ionization and temperature structure of the X-ray illuminated gas and the reprocessed spectrum in the optical, UV, and X-ray ranges. The radiative transfer equation is solved in multi-stream approximation by the Accelerated Lambda iteration (ALI) method for the continuum and for the lines. The resulting ionization and temperature data are then read by the Monte-Carlo code NOAR. It calculates heating and cooling rates due to Compton scattering in the medium and the final emerging X-ray spectra for the reprocessed radiation in the range of $(0.8-100) \mathrm{keV}$. The resulting spectra are computed at 20 different emission angles $\psi$ measured with respect to the disk normal and varying in constant steps of $\cos \psi$.

\subsection{Keplerian motion and relativistic effects}

A distant observer detects an evolving spectrum to which all parts of the orbiting hot spot contribute. This contribution depends on the local spectral emissivity but also on the relativistic effects and on the Doppler shift acting at a given position.

The radiation propagation for a Keplerian accretion disk around a black hole is computed by the code KY (Dovčiak et al. 2004; Dovčiak 2004). In our application, KY integrates the photon geodesics between a position inside the orbiting hot spot and an observer at infinity. The integration method takes into account the energy shift of the photon along the geodesics, arrival timelags, and the lensing-effect. Spectra are computed for various viewing directions, $i$, of a distant observer based on a given timedependent emissivity distribution of the flare spot. We define the initial position of the spot center in polar coordinates $R_{0}$ and $\phi_{0}$. With $h=0.5 R_{\mathrm{g}}$ and $\theta_{0}=60^{\circ}$, the spot radius, $r_{0}$, follows to be $r_{0}=\sqrt{3} h=0.866 R_{\mathrm{g}}$.

The flare above the disk surface is assumed to co-rotate with the underlying disk. This is a natural assumption if the flares form around reconnecting magnetic field lines that are anchored in the disk interior. The spot center follows the same Keplerian orbit. Since the spot originates from external illumination, we can assume that its initial shape remains circular. Nevertheless, in principle the kinematics of the spot is affected by the differential rotation of the disk: spot regions located closer to the black hole have a larger angular velocity than spot regions farther away. Therefore there is a relative drift of the material across the spot, with some material leaving the spot region and cooling off while new material enters and is heated. We assume that at each part of the spot the orbital motion is Keplerian but, for simplicity, we neglect the relative drift of the material and preserve the spot's circular shape We thus suppose that the heating and cooling of the drifting material is instantaneous. This is a safe assumption following the estimates by Collin et al. (2003).

In this paper, we consider the relativistic light bending only for the radiation being re-emitted by the hot spot. Modeling the relativistic effects for the incident radiation is not necessary because the flare occurs at a small height. We checked this approximation conducting ray-tracing computations for incident photons being emitted along the limiting cone of the flare at $\theta_{0}=60^{\circ}$. In flat space, these photons trace out a circle on the surface plane of the disk. For a flare in Schwarzschild metric at radius $7 R_{\mathrm{g}}$ and height $0.5 R_{\mathrm{g}}$, the relativistic trace does not differ significantly from the flat-space result.

\subsection{Time evolution of flare and spot}

The time evolution of the flare is based on two aspects. First, there is an intrinsic time evolution of the flare source, which is parameterized by a specific profile. For simplicity we assume that the intrinsic flare emission has a rectangular shape of length $T$. The source is thus switched on and off instantaneously. Second, there is a delayed response from the various parts of the spot, as the primary radiation emitted by the elevated source reaches each concentric ring of the hot spot consecutively. During onset and fading of the flare, the hot spot evolves from the center, where the first primary photons arrive, to the border. This evolution adds time-dependent effects to the spectrum that are different from those caused by relativity and by the orbital spot motion. Let the irradiation at the spot center $\left(R_{0}, \phi_{0}\right)$ start at time $t_{0}$ and end there at $t_{0}+T$. Then the local emissivity $\varepsilon$ at the photon energy $E$, given disk position $(R, \phi)$, emission angle $\psi$, and time $t$ is determined by:

$\varepsilon(E, R, \phi, \psi, t)=\left\{\begin{array}{cl}\varepsilon_{\mathrm{spot}}(E, R, \phi, \psi), & t_{0}<t-\eta<t_{0}+T, \\ 0, & \text { otherwise },\end{array}\right.$

with $\eta=\left(\sqrt{h^{2}+r^{2}}-h\right) / c$, and the spatial distance between the spot center and the point $(R, \phi)$ is measured by $r=$ $\sqrt{R^{2}+R_{0}^{2}-2 R R_{0} \cos \left(\phi-\phi_{0}\right)}$. The local spot emission as computed from the radiative transfer simulations is denoted by $\varepsilon_{\text {spot }}$. If the duration of the flare is much longer than the light travel time across the spot, the irradiation can be considered as a stationary process and the onset and fading phases are negligible. Such a time-steady hot spot is described by the local disk emissivity

$\varepsilon(E, R, \phi, \psi)=\left\{\begin{array}{cl}\varepsilon_{\text {spot }}(E, R, \phi, \psi), & r<r_{0} \\ 0, & \text { otherwise }\end{array}\right.$

Comparison with the observational data requires relativistic raytracing and time integration of the local spot emissivity while taking into account the orbital motion. These computations are consistently carried out by KY.

\section{The local properties of the reprocessed spectra}

The overall response of the irradiated disk to the incident flux is in agreement with known results: a hot layer forms in the upper disk atmosphere, being at a temperature comparable to the Compton temperature (Raymond 1993; Ko \& Kallman 1994; Nayakshin et al. 2000; Ballantyne et al. 2001; 
Różańska et al. 2002). At larger optical depth a rapid transition to colder, less ionized layers follows. Nevertheless, as we show in the following, the horizontal stratification of the hot spot we introduced in our model brings new aspects to the structure of the medium and to the obtained reprocessed spectra.

\subsection{Temperature profiles and reprocessed spectra}

Figure 2 (top graph) shows the vertical temperature structure of the disk after the onset of the flare. We plot the temperature as a function of the Thomson optical depth and for the medium underneath three of the five concentric spot rings (inner, intermediate, and outer region of the spot). The profiles are plotted versus the Thomson optical depth $\tau_{\mathrm{ES}}$, which increases toward the equatorial plane of the disk. The hot surface layer of the disk atmosphere and the abrupt transition to the colder medium are visible. At the spot center the temperatures are higher, the hot skin is thicker, and the less ionized layers lie deeper in the medium. For a given spot ring, the heated layer at $7 R_{\mathrm{g}}$ is narrower than at $18 R_{\mathrm{g}}$ which is determined by the different density profiles as computed before irradiation by the flare.

The local reprocessed spectra emitted by different spot rings are shown in Fig. 2 (bottom graph). Going from top to bottom, the three panels present results for the inner, intermediate, and outer spot ring respectively. The three intensity spectra of each panel correspond to emission angles of $\psi=20^{\circ}$ (solid line), $\psi=$ $60^{\circ}$ (long-dashed line), and $\psi=80^{\circ}$ (short-dashed line). At the lowest $\psi$, corresponding to a Seyfert- 1 view, the Compton hump over (10-60) keV appears similar for all rings. At higher $\psi$, the normalization of the emitted spectra increases and the spectral slope hardens from the spot center toward the border. For a given ring, the spectral normalization rises with $\psi$ constituting a limbbrightening effect of the hot spot. Such behavior had been first seen in models of irradiated constant density media (Fig. 7 of Życki \& Czerny 1994).

The limb-brightening can be partly understood from the vertical temperature structure of the medium: the upper layers of the disk surface are very hot, therefore highly ionized, and optically thin. A local observer looking at the medium at a normal direction $\psi \sim 0^{\circ}$ could see down to the deeper, less ionized layers, because along this line-of-sight the temperature drops significantly within $\tau_{\mathrm{ES}}=1$. However, a local observer looking at the same medium from a higher inclination $\psi \sim 60^{\circ}$ would rather see the highly ionized surface layers dominated by electron scattering and exhibiting less absorption. Along this line-of-sight the extinction within $\tau_{\mathrm{ES}}=1$ is dominated by scattering and thus more intensity is preserved than for the observer at low inclinations.

We also investigate the dependencies of the reprocessed continuum on $\psi$ by considering spectral intensity ratios over narrow energy bands at $4 \mathrm{keV}$ and at $28 \mathrm{keV}$. The results are plotted in Fig. 3. The plot shows that the spectra become harder toward lower emission angles. This behavior varies quantitatively with the location inside the spot. At the spot center the irradiating flux is strongest and the softest spectra appear. This is true for all possible emission angles and can be understood by the relatively thicker high-temperature layer at the disk surface. It prevents a large fraction of the incident photons from reaching the deeper, colder medium, where they can be absorbed. Their chance to escape from the atmosphere after a few electron scattering events is higher than at the spot border, where the medium absorbs more efficiently.

\subsection{The iron fluorescence complex}

The spectral appearance of the iron K-line complex also varies with $\psi$ and with the position inside the hot spot. Generally, several components of the line complex are visible: one at $6.4 \mathrm{keV}$ representing weakly ionized/neutral iron, the component of helium-like iron at $6.7 \mathrm{keV}$, and sometimes the hydrogenlike iron at $6.9 \mathrm{keV}$. A $\mathrm{K} \beta$-line at $7.1 \mathrm{keV}$ is visible when the weakly ionized component at $6.4 \mathrm{keV}$ is strong. For a given spot ring, the lines emitted at high $\psi$ indicate stronger ionization than at low $\psi$. The effect is analogous to the limb-brightening discussed above and can be again understood from the temperature and ionization structure of the medium.

The iron K-lines and also the soft X-ray emission lines of the local spectra we compute are particularly strong with respect to the continuum. This is partly explained by the fact that we only consider the pure reprocessed radiation and we leave aside any dilution by the primary component. Another reason is that in our model the relatively cold disk matter at roughly $10^{5} \mathrm{~K}$ is irradiated by hard X-rays corresponding to a temperature of $\left(10^{7}-10^{8}\right) \mathrm{K}$. Since we assume that the flare duration is significantly shorter than the dynamical timescale of the medium, the density structure of the disk atmosphere does not change. For longer flare durations, the medium must thermally expand, as shown in Czerny \& Goosmann (2004) and Różańska et al. (2002). During the expansion the density decreases leading to weaker line emission (Collin et al. 2003). This effect can be important for flares completing more than a single orbit, as the one analyzed in NGC 3516 by Iwasawa et al. (2004).

\section{Spectra seen by a distant observer}

To obtain the reprocessed spectra seen by a distant observer, we investigate the effects of the intrinsic time evolution of a flare combined with general relativity and with the orbital motion in the vicinity of the black hole. We apply the ray-tracing code KY with the local single-ring spectra described in Sect. 3 to define the emissivity of the spot. Note that in our modeling we focus on the re-emission of the spot and we do not take into account the primary emission. We thus assume that the flare source is shielded from the observer's direct view and that it manifests itself mainly by its reflection.

\subsection{Persistent spots at different orbital phases}

In a first step, we consider the case when the flare duration is much longer than the light crossing time of the hot spot. We thus neglect the onset and the decay phase of the flare when the spot is only partly illuminated. In the following we refer to this type of flares as persistent flares. To define the local emission we use the time-steady spot approximation (see Eq. (2)). Beside the location of the flare $R_{0}, \phi_{0}, h$, and the size of the spot $\theta_{0}, r_{0}$ given in Sect. 2.3, we assume a flare duration equal to $1 / 8$ of the local orbital period.

\subsubsection{Spectral appearance}

In Fig. 4 we plot the spot spectra of a persistent flare occurring at different orbital phases $\phi_{0}$. The time-integrated spectra over (1-50) $\mathrm{keV}$ are shown for three disk inclinations with respect to a distant observer: $i=30^{\circ}, i=60^{\circ}$, and $i=85^{\circ}$ (top, middle and bottom panels respectively). The impact of general relativity effects and of the spot motion becomes more important with 
R. W. Goosmann et al.: Iron lines from transient versus persistent hot spots
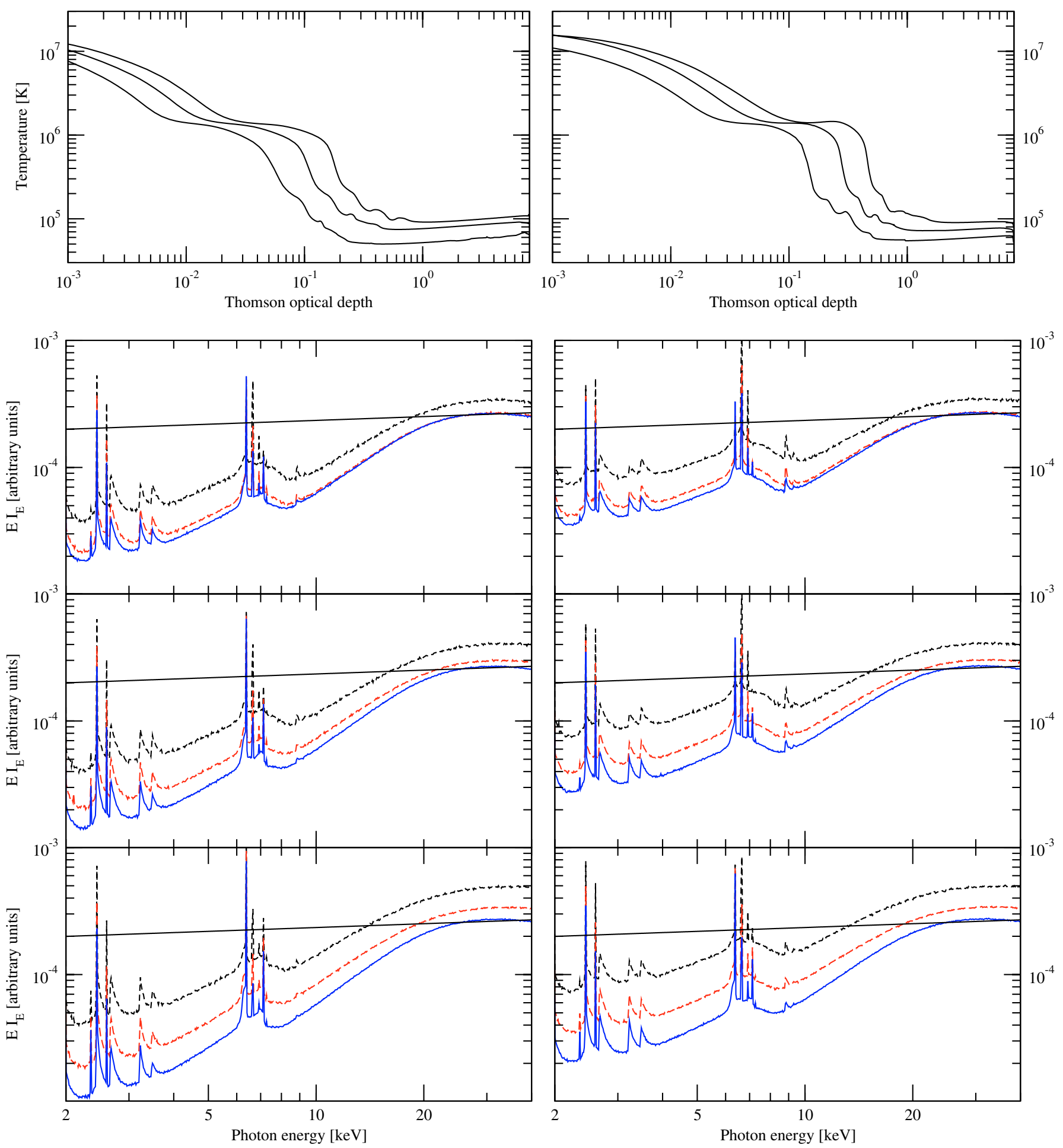

Fig. 2. The top graph shows the vertical temperature profiles for three concentric rings of the hot spot. They correspond to incident angles of $\theta=18^{\circ}$ (inner ring, top curves), $\theta=41^{\circ}$ (intermediate ring, middle curves), and $\theta=57^{\circ}$ (outer ring, bottom curves). In the same order, the three panels of the bottom graph show the corresponding local reprocessed intensity spectra in $E \times I_{\mathrm{E}}$ at three different emission angles: $\psi=20^{\circ}$ (blue, solid), $\psi=60^{\circ}$ (red, long dashes), and $\psi=80^{\circ}$ (black, short dashes). The primary spectrum is indicated by the straight lines. The angles $\theta_{0}$ and $\psi$ are measured with respect to the disk normal. Results for a hot spot at $R=7 R_{\mathrm{g}}(l e f t)$ and $R=18 R_{\mathrm{g}}($ right $)$ are presented.

increasing $i$. It affects the normalization of the reprocessed spectra as well as the shape of specific features. At both distances from the black hole considered here, the relativistic modifications are relevant, but they are more significant at $7 R_{\mathrm{g}}$ than at $18 R_{\mathrm{g}}$, as expected. The spacetime curvature and the Doppler shifts are stronger when being closer to the black hole. At $7 R_{\mathrm{g}}$, the iron K-line complex cannot be properly decomposed into components for any $\phi_{0}$ and $i$. An exception occurs at $i=60^{\circ}$, where shortly "after" the passage "behind" the black hole some structure of the line appears. Still, the deformations remain quite strong also in this case.

The spectral variations of a time-steady spot orbiting around a black hole are not only due to relativistic Doppler shifting and gravitational lensing. To an important extent they are also caused 

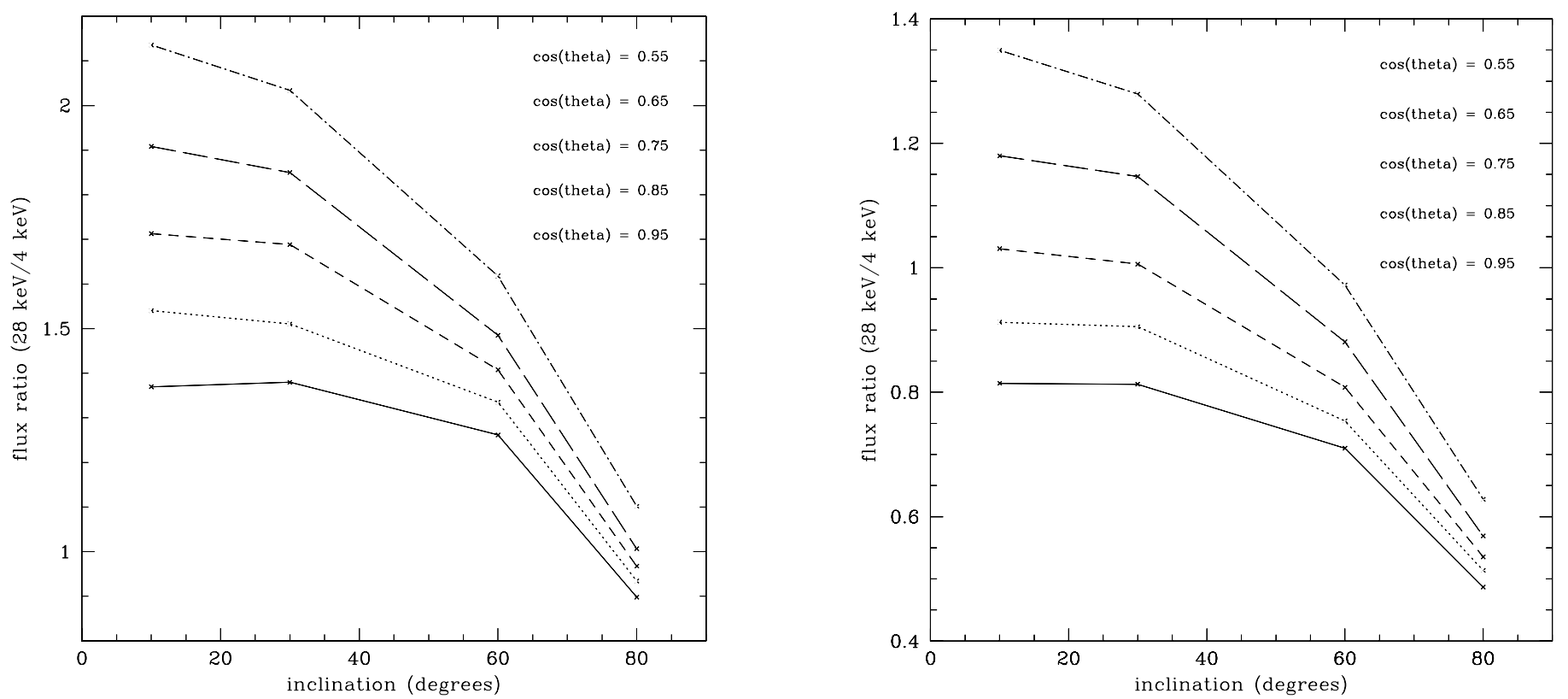

Fig. 3. Spectral intensity ratios between $28 \mathrm{keV}$ and $4 \mathrm{keV}$ plotted versus the local emission angle $\psi$ (local disk inclination) for a hot spot at $7 R_{\mathrm{g}}$ (left) and at $18 R_{\mathrm{g}}($ right $)$. The five curves denote the consecutive concentric rings of the hot spot. They are parameterized by the cosine of the incident angle $\theta$ of the illuminating primary radiation. The angles $\theta$ and $\psi$ are measured with respect to the disk normal.

by time delay effects that are due to variations in the "length" of different photon geodesics reaching the observer at a given time in his/her reference frame (Dovčiak et al. 2007). Gravitational lensing is especially important at edge-on viewing directions, when the spot, the black hole, and the observer are (nearly) lined up. At $i=85^{\circ}$, the maximum of the spectrum therefore occurs when the spot passes "behind" the black hole, at $\phi_{0}=180^{\circ}$ (top middle box in each panel). At intermediate inclinations the Doppler shifting is more dominant. In contrast to the Newtonian case, the maximum (minimum) relativistic Doppler shift does not occur when the spot is approaching (receding from) the observer at maximum velocity; it rather appears around $\phi \sim 225^{\circ}$ $\left(\phi \sim 45^{\circ}\right)$, which agrees with the results shown in Fig. 4.

\subsubsection{Impact of the spot structure and of the angle-dependent local emission}

We investigate the impact of the horizontal spot structure and the angle dependent emission on the results. Therefore we compute time-integrated, relativistically convolved spectra for the following three cases of the local spot emission:

a) including the detailed horizontal spot-structure and the $\psi$-dependence of the local spot emission,

b) averaging over the horizontal spot structure but keeping the dependence on $\psi$,

c) averaging over the horizontal spot structure and over $\psi$.

We use the same model setup as for Fig. 4 and we define the specific case of $\phi_{0}=225^{\circ}$, i.e. the spot is on the approaching side of the disk at the orbital phase where the strongest relativistic modifications are expected. The spot then proceeds up to $\phi=270^{\circ}$. The inclination of the observer is set to $i=60^{\circ}$. This may correspond to a maximum type- 1 viewing angle for very luminous AGN (quasars).

We obtain the following results: the differences between a horizontally structured spot and a uniform one are negligible. The spot structure leaves a trace in the spectral evolution only during the rising and fading phases of the flare. These phases are very short compared to the total flare life-time. The timeintegrated spectrum is therefore dominated by the reprocessed radiation coming from the fully illuminated spot, which evidently can be well approximated by uniform emission. Accurate computations of the angular emissivity dependence are slightly more important since they significantly affect the normalization of the reflected component. Nevertheless, the spectral shape is not strongly influenced: when the spectra are renormalized, the differences between the cases a) and c) are less than $7 \%$ at all energies.

We conclude that for a spot seen at $i=60^{\circ}$ and orbiting at $R=7 R_{\mathrm{g}}$ it is safe to simplify the computations for persistent flares by averaging over the spot expansion. The same holds for lower inclinations, for other orbital phases than $\phi_{0}=225^{\circ}$, and for higher values of $R$ because in all these cases the impact of the relativistic and Doppler effects is smaller than for our test case.

\subsubsection{Detecting the orbital phase of a revolving spot}

In recent years, observations with $X M M-N e w t o n$ have started to reveal the radial irradiation structure of the innermost regions in AGN accretion disks. For the Seyfert galaxy NGC 3516 Iwasawa et al. (2004) reported time-dependent spectral data that are in agreement with the modeling of an orbiting hot spot between $7 R_{\mathrm{g}}$ and $16 R_{\mathrm{g}}$. The data suggest a periodic signal in the iron line flux, which can be interpreted as a long-term flare completing several orbits. Nevertheless, the sensitivity of XMM-Newton is not sufficient to clearly resolve sub-orbital features in the spectral evolution of NGC 3516. Such investigations, which allow to map out the azimuthal irradiation structure of the inner accretion disk, will become possible with the much higher effective collecting areas of future satellite observatories like XEUS and Constellation- $X$.

To illustrate the spectral appearance of sub-orbital X-ray signals we simulate the observation of an orbiting hot spot applying our persistent flare model. We adopt the response matrices 

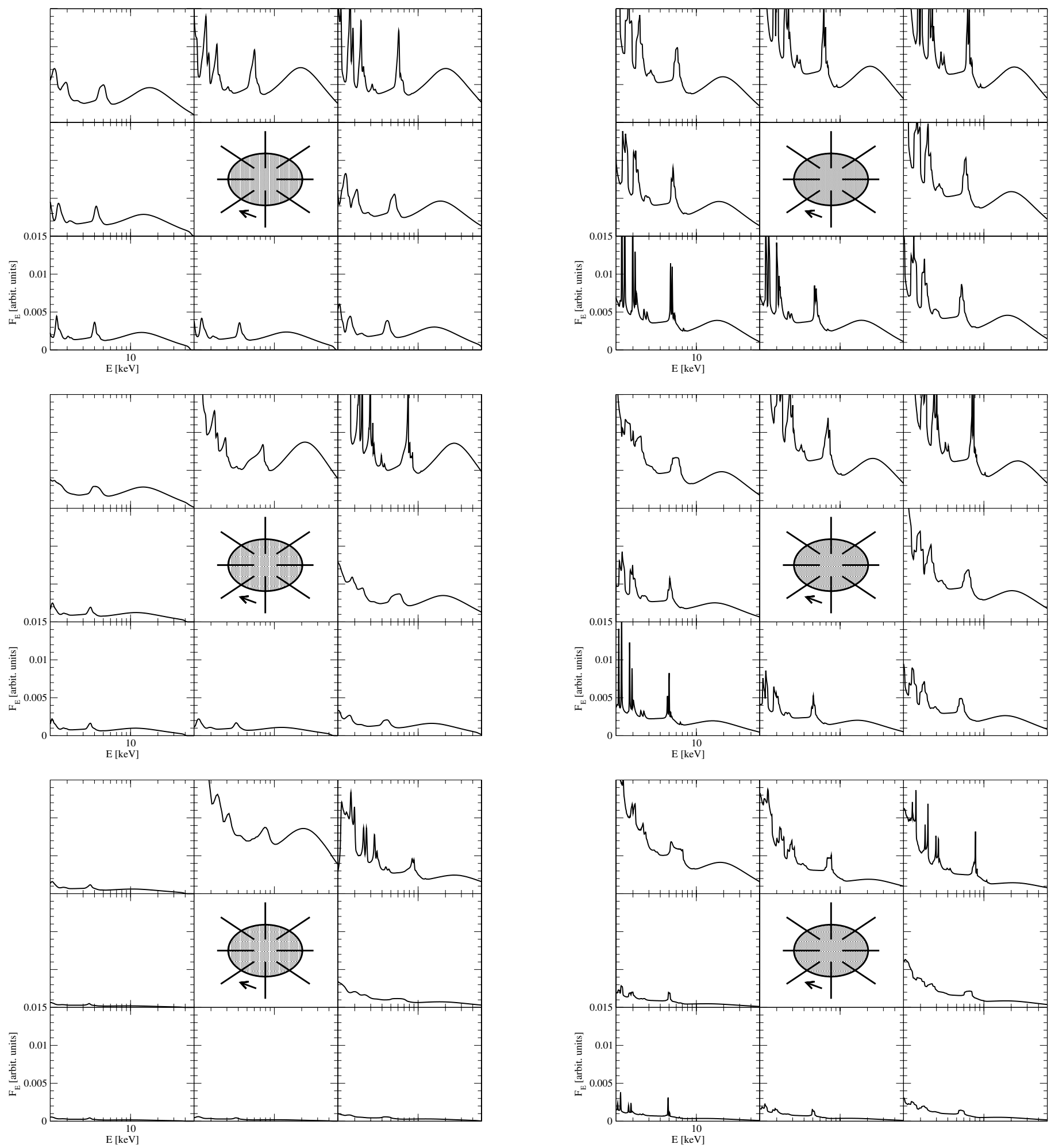

Fig. 4. Spectra emitted by a persistent hot spot at 8 different orbital phases. In each panel the bottom middle spectrum corresponds to the starting phase of the spot's closest approach to the observer $\left(\phi_{0}=0^{\circ}\right)$. The spot exists for $1 / 8$ of the orbital time scale at its location and the disk rotates clock-wise. From top to bottom the panels represent different viewing angles, $i$ (measured from the disk normal): $i=30^{\circ}, i=60^{\circ}$, and $i=85^{\circ}$. The spot is located at a distance of $7 R_{\mathrm{g}}($ left $)$ and $18 R_{\mathrm{g}}($ right $)$ from the black hole.

of the XEUS TES detector as they are available in April 2007 and of the EPIC PN camera aboard XMM-Newton. The simulations for both satellites are conducted in XSPEC V.11.3.2 using a modified version of the KYLCR model (Dovčiak et al. 2004). The model allows to implement our local spot spectra described in Sect. 3 after they are averaged over the horizontal spot expansion. According to Sect. 4.1.2, this simplification does not induce spurious effects. With KYLCR we compute the relativistically blurred, time-integrated spectrum of the spot that we use to produce the simulated XEUS data. We define a persisting spot lasting for $1 / 8$ of an orbit at $R=7 R_{\mathrm{g}}$ on the receding $\left(\phi_{0}=45^{\circ}\right)$ and on the approaching $\left(\phi_{0}=225^{\circ}\right)$ side of the disk. We 

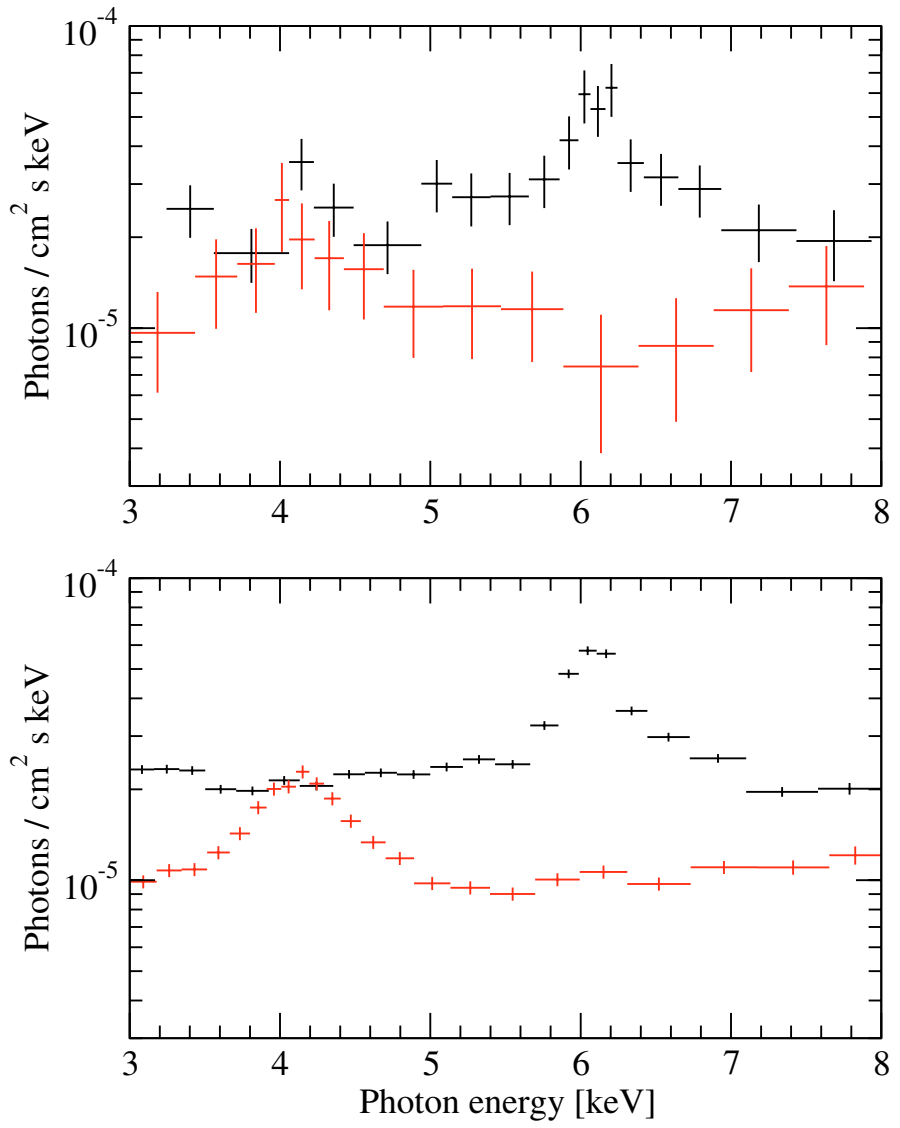

Fig. 5. Simulated observations by XMM-Newton (top) and XEUS (bottom) of a relativistically broadened iron K-line emitted by localized $\mathrm{X}$-ray sources that orbit at $7 R_{\mathrm{g}}$ around a $10^{8}$ solar mass black hole. The two hot spots are at opposite positions, on the receding $\left(\phi_{0}=45^{\circ}\right.$, red) and the approaching $\left(\phi_{0}=225^{\circ}\right.$, black) side of the accretion disk.

assume a Schwarzschild metric and a disk inclination of $i=30^{\circ}$. The simulated photon flux of $5 \times 10^{-5}$ photons $/\left(\mathrm{s} \mathrm{cm}^{2} \mathrm{keV}\right)$ in the variable red wing of the iron line complex is adjusted to the observation of NGC 3516 analyzed in Iwasawa et al. (2004). We assume an observation time $T=7300 \mathrm{~s}$, which corresponds to the flare life time assuming a black hole of $10^{8}$ solar masses. The fake data are rebinned using the tool GRPPHA to ensure that each bin contains a minimum of 50 (200) counts for the XMM-Newton (XEUS) "simulation".

In Fig. 5 we plot the produced spectra. For XEUS the flares on the two sides of the accretion disk can be distinguished from each other as the normalization and the position of the iron Kline differ significantly. Also in the XMM-Newton spectrum these differences are visible but the much higher noise level does not allow the same clear conclusion. Note that in these simulations we assume that the primary radiation does not dilute the reflection. This holds true only if the object is strongly reflectiondominated or if the primary radiation has been precisely removed in the data analysis. In the latter case additional errors are expected, which makes it even less likely that XMM-Newton resolves such sub-orbital features.

\subsubsection{Probing the local spot spectrum}

The computations of the local spectra we use include the vertical disk structure and the radiative transfer in such a stratified medium. The resulting reprocessed spectra reveal several
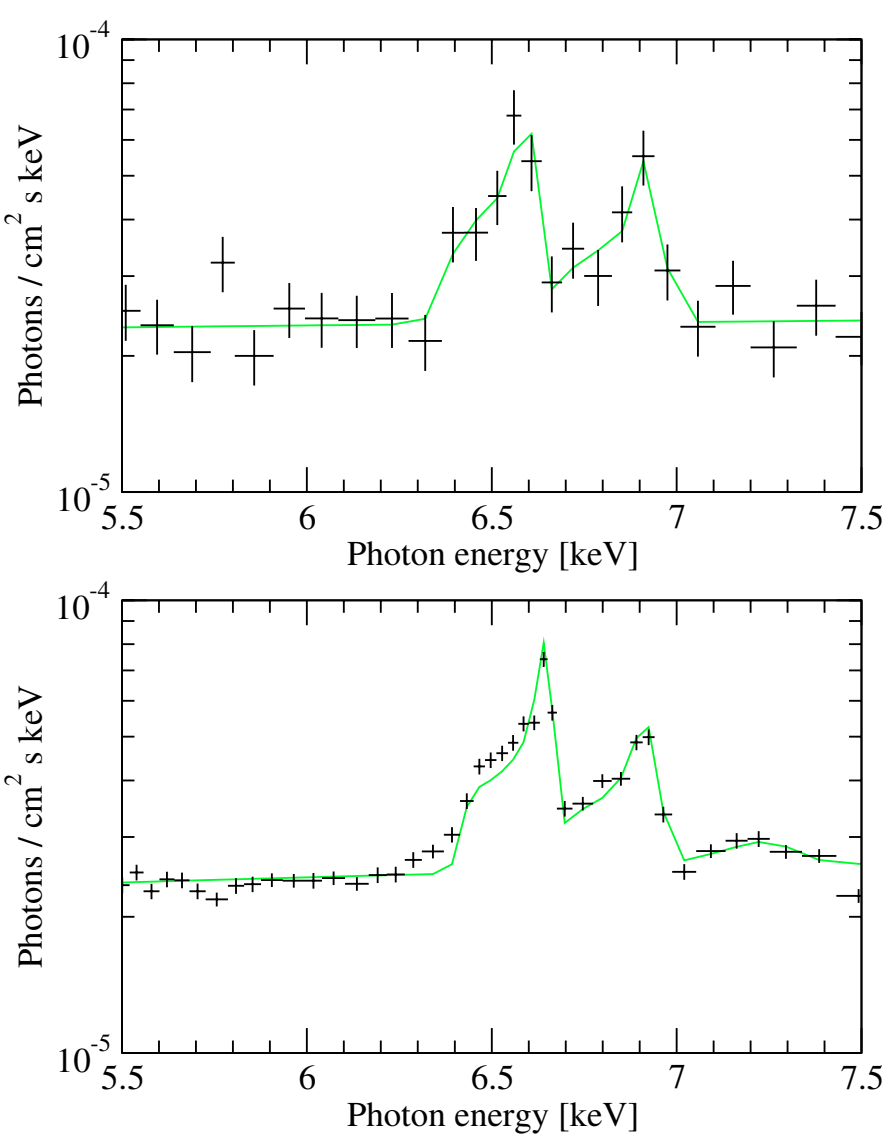

Fig. 6. Best fit for the simulated XMM-Newton (top) and XEUS (bottom) data of an approaching hot spot orbiting at $18 R_{\mathrm{g}}$ around a $10^{8}$ solar mass black hole. The best fit models are indicated by solid lines: "power law +2 KYGLINE components" for the XMM-Newton data and "power law + 3 KYGLINE components" for the XEUS simulations.

iron line components, which are then blurred by the relativistic effects. The subtleties of the resulting spectrum cannot be well resolved in present-day X-ray data, and the question arises whether they will be visible with more advanced instruments in the future. We investigate to which extend the spectral structure in the iron line band can be observationally resolved by comparing simulated data from XMM-Newton and XEUS for a persistent flare.

We consider a hot spot lasting for $1 / 8$ of the orbital period at $R=18 R_{\mathrm{g}}$ corresponding to an integration time of $30700 \mathrm{~s}$ for a $10^{8}$ solar mass black hole. At this distance radius from the black hole, the relativistic effects are still relevant but not as violent as close to the marginally stable orbit. We therefore expect to still detect some structure of the iron line in the fake observations. The flare is supposed to start on the approaching side of the accretion disk, at $\phi_{0}=225^{\circ}$, and the system is seen at an inclination of $i=30^{\circ}$. The simulated observations are then obtained as described in Sect. 4.1.3. They are shown in Fig. 6.

For the purpose of data analysis, it is a common contemporary approach to relativistically blur one or several narrow Gaussians to approximate the local profile of the iron line, whereas the continuum is modeled by an underlying power law (which is invariant under the relativistic effects). We investigate how well the simulated data of a persistent spot can be reproduced by such phenomenological models. We fit the simulated data consecutively adding more relativistically broadened line components to the underlying continuum power law. The lines 
Table 1. Best-fit parameters for simulated XEUS and XMM-Newton observations of a hot spot at the phase of approaching motion. Power law models combined with up to three relativistically blurred iron lines are compared.

\begin{tabular}{llccc}
\hline \hline Model component & Parameter & XEUS & XMM-Newton & XMM-Newton \\
\hline Power law & $\Gamma$ & -0.303 & -0.112 & -0.091 \\
& $N$ & $1.42 \times 10^{-05}$ & $1.91 \times 10^{-05}$ & $1.96 \times 10^{-05}$ \\
KYGLINE \#1 & $E_{\mathrm{c}}[\mathrm{keV}]$ & 6.381 & 6.340 & 6.458 \\
& $\sigma[\mathrm{eV}]$ & 12.713 & 20.243 & 180.95 \\
& $N$ & $6.06 \times 10^{-06}$ & $6.44 \times 10^{-06}$ & $1.09 \times 10^{-05}$ \\
\multirow{3}{*}{ KYGLINE \#2 } & $E_{\mathrm{c}}[\mathrm{keV}]$ & 6.647 & 6.659 & - \\
& $\sigma[\mathrm{eV}]$ & 21.931 & 0.115 & - \\
& $N$ & $4.19 \times 10^{-06}$ & $4.35 \times 10^{-06}$ & - \\
KYGLINE \#3 & $E_{\mathrm{c}}[\mathrm{keV}]$ & 6.983 & - & - \\
& $\sigma[\mathrm{eV}]$ & 59.741 & - & - \\
& $N$ & $8.85 \times 10^{-07}$ & - & - \\
\hline & & $352 / 250$ & $27 / 33$ & $39 / 36$ \\
\hline
\end{tabular}

are implemented by the KYGLINE model that is also available in XSPEC. The disk emission pattern is defined exactly as for the data simulations, but the parameters of the power law as well as the line centroid, width, and normalization are allowed to vary in the fitting process. The fitting is restricted to the spectral range of (4.0-7.5) keV to avoid the soft X-ray features and the strong iron absorption edge.

As expected the resulting phenomenological fits are much better determined for the more precise XEUS data than for the $X M M-N e w t o n$ simulations (Fig. 6). For XEUS it is possible to find a phenomenological fit for three distinguished components of the $\mathrm{K} \alpha$-line complex (Table 1): neutral/weakly ionized iron $(\sim 6.4 \mathrm{keV})$, helium-like iron $(\sim 6.7 \mathrm{keV})$, and hydrogenlike iron $(\sim 6.9 \mathrm{keV})$. The attempt to also identify an individual $\mathrm{K} \beta$-line around $7.1 \mathrm{keV}$ fails, instead the hydrogen-like component seems particularly broad indicating a possible blend. All line centroids are slightly redshifted with respect to the laboratory centroid energies, also the hydrogen-like $\mathrm{K} \alpha /$ neutral $\mathrm{K} \beta$ line when interpreted as a blend. Visually the residuals imply that the fit misses some curvature in the spectrum red- and bluewards of the broadened iron line. The intrinsic spectral shape across the iron-line band is more complex than a power law continuum plus relativistically blurred Gaussian lines can account for. In the local reprocessing spectra, the various components of the iron K-line complex seem to stand on a "pedestal" between $6 \mathrm{keV}$ and $7 \mathrm{keV}$ (see Fig. 2). This broad feature is mainly due to the drop in continuum normalization at the iron $\mathrm{K}$ absorption edges above $7.1 \mathrm{keV}$. The edges should therefore be considered in the fitting. Redward of the $6.4 \mathrm{keV}$ line, some impact of a Comptonized line shoulder might also be relevant.

In the case of XMM-Newton, the number of required subcomponents is much less well-defined (Fig. 6). Models with one or two Gaussian components both lead to acceptable fits (Table 1), but including both, a neutral/weakly ionized and a helium-like $\mathrm{K} \alpha$-component deliver the slightly better fit. For the model that only consists of one line the line width is very large as expected. In the two-line model only the neutral/weakly ionized iron line is broad and the helium-like component appears very narrow. Any further line around (6.9-7.1) keV remains undetected.

An important result of the modeling is, however, that repeating the same data simulations for XMM-Newton shows a significant uncertainty in the fitting results and their interpretations. The results for XEUS on the other hand are stable. This documents that the sensitivity of XMM-Newton is not high enough to draw reliable conclusions about the sub-structure of the broad iron line shape coming from a persistent flare at $18 R_{\mathrm{g}}$ as it is considered here.

\subsection{Transient spots at different orbital phases}

In a second step we investigate the reprocessed spectra of flares with a life-time that is comparable to the light-crossing time of the spot. We name such spots as transient and we investigate their emission at defined orbital phases. The irradiating primary emission is switched on and off instantaneously. The emission lasts for one gravitational time scale, $T_{\mathrm{g}}=\frac{G M}{c^{3}}$, which compares to the spot's light-crossing time by $T_{\mathrm{g}} \sim 1.15 \frac{r_{0}}{c}$. During $T_{\mathrm{g}}$ the spot proceeds by the azimuthal angle $\Delta \phi(R)=\left(R_{\mathrm{g}} / R\right)^{1.5}$. At the two disk radii considered here this amounts to $\Delta \phi\left(7 R_{\mathrm{g}}\right) \sim 3^{\circ}$ and $\Delta \phi\left(18 R_{\mathrm{g}}\right) \sim 0.75^{\circ}$. Hence, during the flare irradiation the spot remains nearly at the same orbital phase. For the initial azimuthal position $\phi_{0}$ of the flare we consider the values $\phi_{0}=0^{\circ}$, $\phi_{0}=90^{\circ}, \phi_{0}=180^{\circ}$, and $\phi_{0}=270^{\circ}$. We use the same radiative transfer solutions for the local reprocessed spectra as in Sect. 3. We then run KY to compute sets of relativistically blurred, timedependent spectra at the viewing angles $i=30^{\circ}, i=60^{\circ}$, and $i=85^{\circ}$.

\subsubsection{Spectral appearance}

In Fig. 7 we present the obtained integrated spectra for the observed flare periods and the corresponding lightcurves at the different azimuthal positions. The observed flare durations are of the order of a few hundreds of seconds. The plots show that line broadening is important also for transient flares. But in contrast to the persistent spots the spot velocity projected along the observer's line-of-sight does not change significantly during the flare period; this holds true even at high disk inclinations as the spot only moves by very small values of $\Delta \phi$. We therefore conclude that the line broadening is caused to a large extent by general relativity. Due to the expansion of the spot, the photons arriving at the distant observer at a given time originate at different positions on the accretion disk. Hence, their gravitational redshift, which only depends on the distance of the emission point from the black hole, is distributed over a certain range and the line appears to be broadened. At $18 R_{\mathrm{g}}$ (right panels of Fig. 7) the gravitational line broadening is weaker and the iron K-line complex is resolved into its sub-components at all orbital phases. When comparing the iron K-lines for the same $\phi_{0}$ but at different inclinations, the effects of the vertical ionization and temperature profile of the disk become visible (see Sect. 3) and indicate that toward higher inclinations the medium appears to be more strongly ionized.

Some aspects of the spectral shape are consistent with the results obtained in Sect. 4.1 for a persistent hot spot completing a significant fraction of the orbit. The Doppler shift of the $\mathrm{K} \alpha$ line complex, for instance, corresponds to the orbital phase of the flare. It increases with higher inclination due to a larger velocity component projected along the line-of-sight. For a flare occurring "behind" the black hole, i.e. for $\phi_{0}=180^{\circ}$, the lensing effect strongly modifies the spectrum and increases the overall flux. At this phase the iron K-line is entirely smeared out when seen at high inclinations.

\subsubsection{Lightcurves}

In our modeling of transient flares we consider the different light-travel times between the elevated source and various 

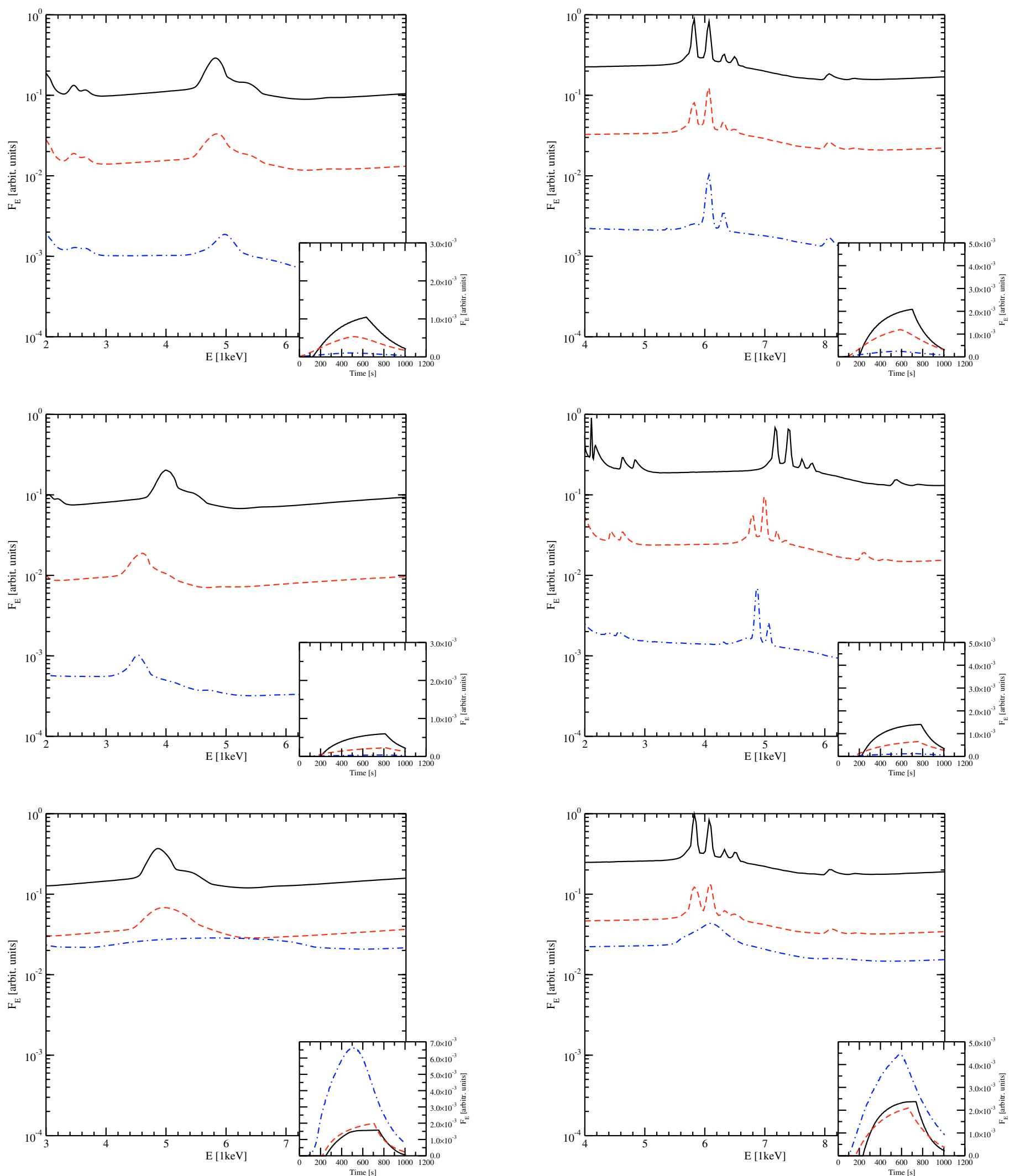

Fig. 7. Integrated flux spectra and lightcurves for short-term flares at four different initial azimuthal positions of the spot. From top to bottom, the panels represent $\phi_{0}=0^{\circ}, \phi_{0}=90^{\circ}, \phi_{0}=180^{\circ}$, and $\phi_{0}=270^{\circ}$ (continued on the next page). The origin of the azimuthal scale at $\phi=0^{\circ}$ is at the closest approach of the hot spot to the (inclined) observer; for $\phi=180^{\circ}$ the spot is farthest away and "behind" the black hole. The spectra were integrated over the entire flare period. The various curves of one panel denote the disk inclinations $i=30^{\circ}$ (black, solid), $i=60^{\circ}$, (red, dashed), and $i=85^{\circ}$ (blue, dot-dashed). The spectra contain a systematic offset by a factor of 5 between each other for clarity. Results for a spot at $R=7 R_{\mathrm{g}}$ (left panels) and at $R=18 R_{\mathrm{g}}$ (right panels) from the central black hole are shown. 


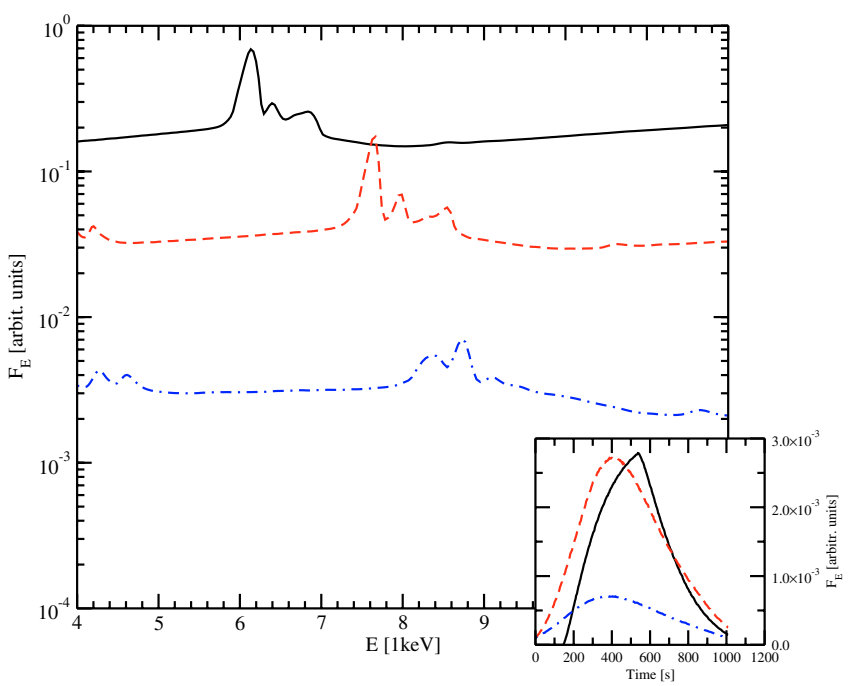

Fig. 7. continued.

positions inside the hot spot. The effect can be seen in the lightcurves shown in Fig. 7. The lightcurves represent the integrated flux between $2 \mathrm{keV}$ and $50 \mathrm{keV}$. The observed flare period is not the same for different values of $i$ and $\phi_{0}$. Physically, it depends on the difference between the "longest" and the "shortest" photon geodesics connecting the spot with the distant observer at a given time in the observational frame. The geodesics are a function of the spacetime geometry and the photon flux they transfer depends on the time-dependent emission across the spot.

At low inclinations the lightcurves have a characteristic and regular shape showing the rising and fading of the flare emission. The shape is not at all box-like as assumed for the incident radiation illuminating the spot. The geometrical expansion of the spot induces different time-delays for the reprocessed radiation and so the onset and the fading of the spot emission are smoothed out. A significant time asymmetry is seen in most cases, with a shallower rise and a more rapid decay. Going toward higher inclinations, the shape of the light curve changes and generally becomes more symmetric - with an exception for the phase $\phi_{0}=180^{\circ}$, when the spot passes "behind" the black hole. The closest symmetry is reached at $\sim 60^{\circ}$. For even larger $i$ the flux decreases rapidly and the lightcurves become much flatter.

\subsubsection{Spectral time evolution}

To investigate the spectral evolution during a short flare we present in Fig. 8 snapshots of a time-dependent spot spectrum. For the particular model shown we consider a spot passing "behind" the black hole $\left(\phi_{0}=180^{\circ}\right)$ and a disk inclination of $30^{\circ}$, which is typical for type-1 AGN.

The development of the flare emission across the hot spot is visible in the spectral sequence. The irradiation sets on at the spot center where it produces the highest temperature and ionization states. Therefore, the iron K-line complex shows stronger components of helium- and hydrogen-like iron for the rising branch of the lightcurve. During the flare period these components become weaker and the $\mathrm{K} \alpha$ and $\mathrm{K} \beta$ emission of less ionized/neutral iron increases. The development of the line components is more clearly visible for a spot at $R=18 R_{\mathrm{g}}$. At $7 R_{\mathrm{g}}$, i.e. close to the marginally stable orbit, the relativistic broadening of the line makes it difficult to distinguish between its individual components. Nevertheless, a development of the line from the onset to the decay of the flare can be seen. The spot evolves

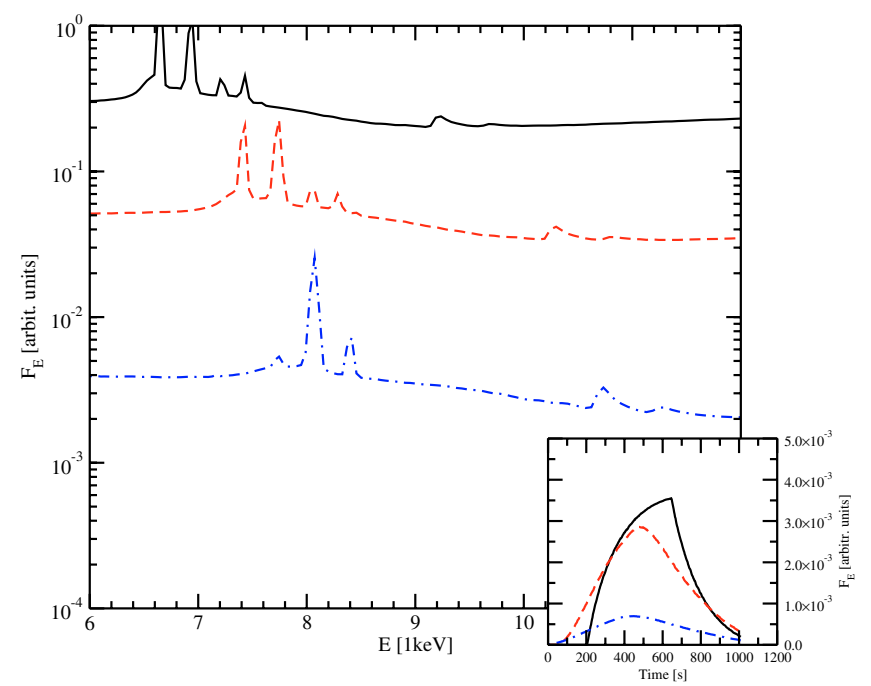

from a small emission site at its center to a circle/ring with outer radius $r_{0}=0.866 R_{\mathrm{g}}$. Hence, the gradient in gravitational and Doppler shifting across the illuminated spot increases during the spot's life-time, so that the width and the centroid of the iron K-line evolve.

\section{Importance of the studied effects}

The spot model we present in this paper contains accurately computed local spectra for the reprocessed flare emission. We include the vertical stratification of the accretion disk and the geometrical details of the flare reprocessing before applying the relativistic modifications and computing the observed spectra. In previous work, these effects have not all been considered. We discuss in this section their impact on the results and we estimate to which extend it is necessary to include them into the modeling.

\subsection{Local emission from the spot}

We calculated the local spot spectrum taking into account the vertical density stratification of the disk. Our local spectra exhibit more than one strong component of the iron line and a complex reprocessed continuum. The continuum component includes a series of iron $\mathrm{K}$ absorption edges above $7.1 \mathrm{keV}$ and a Comptonized shoulder on the red side of the $\mathrm{K} \alpha$-line at $6.4 \mathrm{keV}$. These features give rise to a broad continuum "pedestal" carrying the emission lines, which is not completely smeared out by the relativistic effects even close to the marginally stable circular orbit of a Schwarzschild black hole.

From simulating future XEUS observations of persistent flares in $\mathrm{AGN}$ at $18 R_{\mathrm{g}}$, we conclude that the sub-structure of the iron line complex can be investigated probing the reflecting medium. Using a detailed reflection model for the data analysis instead of phenomenological models has the advantage that the particular spectral characteristics are consistently accounted for. The normalization of the emission lines with respect to the continuum stays on physical grounds and can be connected to global parameters of the system (e.g. ionization parameter, disk radius). For the case of Suzaku observations of AGN the Compton hump around $30 \mathrm{keV}$ can be fitted coherently with the broad iron K-line complex as both features are due to reprocessing (e.g. George \& Fabian 1991). 

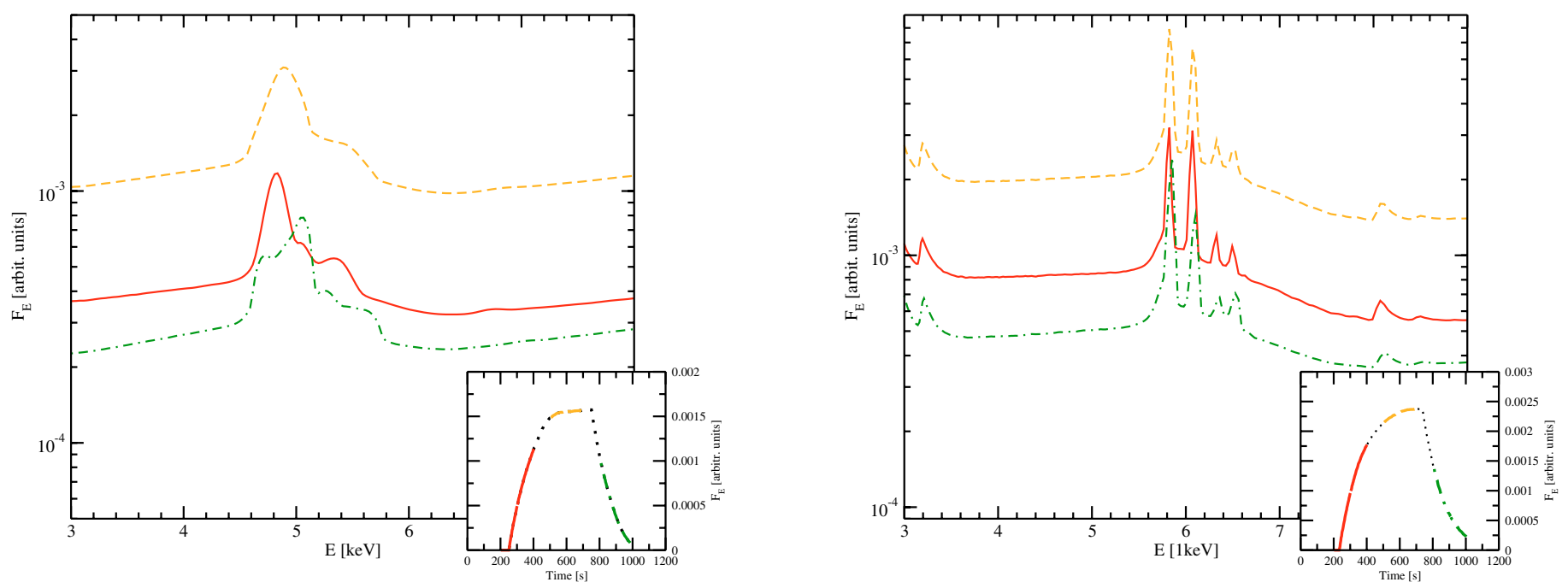

Fig. 8. Spectral evolution and lightcurves for a transient flare located "behind" the black hole $\left(\phi_{0}=180^{\circ}\right)$ with respect to the observer. The observer's inclination is $i=30^{\circ}$. The flare periods are divided into separate time intervals, over which the spectra are integrated. The corresponding time interval of each spectrum is marked on the flare lightcurve. Results for a spot at $R=7 R_{\mathrm{g}}(l e f t)$ and $R=18 R_{\mathrm{g}}($ right $)$ are shown.

Careful computations of the radial structure of a spot are not necessary if the time integration of the data is longer than the light travel time across the spot. In this case the spot spectrum can be approximated as a uniform (average) spectrum which simplifies the calculations. Since the flares considered in the current paper are located at a small height above the disk, the spot radii are small and this assumption is likely to be satisfied in most cases. The radial spot structure is going to be more important for flares located at large heights above the disk, but in this case the flare is a non-local phenomenon, and both the disk radial structure as well as the light bending between the flare and the disk surface might be important. Such a model becomes essentially identical to the lamp-post geometry, which is discussed in Sect. 5.3.

\subsection{General relativity and Doppler effects}

We have included photon energy shifts due to Doppler and general relativity effects, as well as light bending and mutual time delays that influence the observed signal. These effects have a strong impact on the observed spectral features and should be taken into account when modeling reprocessed radiation at distances smaller than $20 R_{\mathrm{g}}$ from the black hole. We find, that the relativistic effects do not only affect the observed shape of the iron K-line emission but also the underlying continuum "pedestal" that appears in the iron line band (see Sect. 5.1).

The relativistic effects are important for the localization of the hard X-ray emission. Short exposure time spectra, which cover only a fraction of the Keplerian timescale of the corotating flare, clearly exhibit the dependency of the observed spectral shape on the spot position. The observer's viewing angle is also important, as it determines the line-of-sight motion of the disk material, i.e. the Doppler shifts. At very high inclinations the gravitational lensing dominates the relativistic effects at the moment when the hot spot passes behind the black hole. This can be a relevant case for Compton-thin type-2 AGN.

\subsection{Flare model versus lamp-post model}

The flare model is a viable scenario for the primary X-ray emission in objects with relativistically broadened iron lines.
Multiple flares are expected if the dynamo action leads to the occasional emergence of magnetic loops filled with plasma above the disk surface. Another popular model assumes that a single variable source is located near the symmetry axis; it could be tentatively identified with a shock at the jet base (Henri \& Pelletier 1991). The existence of a single "lamp post" implies that the loop formation is suppressed and a collimated outflow is considered instead. The two schemes differ rather deeply in the underlying physics (Martocchia et al. 1996, 2000; Miniutti \& Fabian 2004).

Both geometries currently have observational support. A single source at variable heights is able to explain the apparent lack of variability of the line expected to accompany the variations of the primary (Miniutti \& Fabian 2004; Larsson et al. 2007; but see Życki \& Niedzwiecki 2006, for a critical analysis of the model). The multiple flare scenario is supported by detections of powerful irradiation occasionally illuminating the disk at large radii (Turner et al. 2006), and in the case of many co-existing flares the scenario can also explain the apparent suppression (Czerny et al. 2004) and the relative lack of variability (Goosmann et al. 2006a) of the iron K-line.

Obviously the two geometries share common properties, if a lamp-post source is off-axis (Dabrowski \& Lasenby 2001), but differences should be detectable in their time-dependence. The light traveling times between the flare source and the reprocessing disk are significantly larger for a lamp-post geometry than for the flare geometry assumed in this paper. Such delays can be constrained from cross-correlation analysis between the lightcurves at different energy bands as shown in Goosmann et al. (2007). Furthermore, we have shown in Sect. 4.1.3, how the more accurate spectroscopic observations expected from future satellites such as XEUS and Constellation- $X$ will enable to map out the azimuthal details of the irradiated accretion disk the azimuthal pattern of the disk re-emission depends on the radial position of the flare source, and is symmetric only when the flare is located on the disk axis, i.e. in the strict lamp post case.

\section{Discussion and conclusions}

We present exemplary results from very advanced timedependent modeling of the reprocessed emission by a hot spot 
created underneath a magnetic flare. We attempt to test the sensitivity of these models to various simplifying assumptions. This gives the basis to determine a suitable grid of local solutions, which is needed to model an arbitrary flare with reasonable accuracy and efficiency, having in mind future X-ray missions like $X E U S$ and Constellation-X. Our spot model includes the timedependent effects that are caused by the delayed reprocessing from various parts of a spot. We have also included the horizontal spot structure and the angular dependence of the local emission. The local emission is modeled taking into account the vertical disk structure and the observed time-dependent spot spectrum contains all relativistic effects. We summarize the most relevant results we obtain:

- The vertical temperature profile of the disk underneath the hot spot and the resulting local reprocessed spectra vary significantly across the spot surface. The ionized skin of the disk is thicker and the spectrum softer at the spot center than at the spot border. The different components of the iron K-line also follow this temperature gradient.

- At all positions inside the spot, the local spectra show a limb-brightening effect and spectral softening with increasing emission angles $\psi$. The iron line complex develops toward higher ionization states as $\psi$ rises.

- The relativistically modified spectra seen by a distant observer depend significantly on the flare position. As expected, the spectral distortion of the iron K-line becomes more important at smaller disk radii and at higher disk inclinations.

- For persistent flares the radial dependence of the local spectra across the hot spot can be safely averaged over, but the dependence of the local emission angle still has a small impact on the resulting spectrum seen by a distant observer.

- Current observational techniques are only at the threshold to determine the azimuthal phase of persistent hot spots. Their detection would require a perfect subtraction of an eventual primary component.

- Transient spots show a slight spectral development reflecting the evolution of the local emission from the spot center toward the border. They reveal a large variety of shapes in $\mathrm{X}$-ray lightcurves depending on the spot position and the observer's inclination.

Especially the horizontal time-dependent re-emission of the transient spot has not been assessed in previous papers. It turns out that it has a significant impact on the observed lightcurve of the reprocessed radiation. The rising and the decaying phase have characteristic shapes and they do not look symmetric at disk inclinations smaller than $60^{\circ}$. This result will be testable by future observation techniques with a high-resolution and a high time-throughput. On the other hand, we obtain only minor differences in the spectral time-evolution between the rise and the decay of the spot emission. Even with XEUS or Constellation$X$ they should remain undetected as the maximum observation time is set by the flare duration. In our case we assume it to be $1 T_{\mathrm{g}}$, equating only a few hundreds of seconds for a black hole with $10^{8} M_{\odot}$.

The relevant time-scale of a transient flare as we assume it in this work scales with the black hole mass. One could therefore imagine that the spectroscopic analysis of transient flares should become possible for higher black hole masses observed in some quasars. But unfortunately, high mass quasars tend to be more distant and therefore their count rate in the X-ray range is low compared to nearby, lower-mass Seyfert galaxies. Longer observable delay times between the primary and the reprocessed radiation are rather expected when the flare source is located higher above the disk than $H=0.5 R_{\mathrm{g}}$ as assumed in our model. But such a modeling approach requires to also include the relativistic effects for the intrinsic radiation, which could be neglected for the small value of $H$ we considered.

The situation is much more promising for persistent flares. Using XEUS or Constellation- $X$ to observe persistent spots in AGN the obtained data will be accurate enough to constrain not only the azimuthal location from X-ray spectroscopy (see Sect. 4.1.3), but it can even give some hints on the properties of the local spectrum. We find that the horizontal spot structure has practically no impact on the observed spectrum so that the spot can be approximated by a uniform average emissivity. Nevertheless, this simplification would no longer be allowed for spots being close to the marginally stable orbit of a rotating black hole. In this case the relativistic modifications are more extreme and vary more strongly across the spot surface.

In our current model, we have restricted ourselves to the case of flares that occur near above the disk $\left(h \sim 0.5 G M / c^{2}\right)$ and relatively far from the black hole $\left(r \gtrsim 6 G M / c^{2}\right)$, so that the lightbending of the primary X-rays can be neglected. The primary reason for this restriction was motivated by our goal to concentrate on the properties of the spot spectra in short-exposure observations. This restriction means that we do not consider the influence of the black hole rotation, which would further complicate the analysis. Of course, a generalization to the rotating (Kerr) case should be explored in the future, because there are good chances that short-exposure spectra will remove the degeneracy which affects time-integrated spectral profiles. (There are no principal difficulties to include black hole rotation and our suite of numerical codes - TITAN/NOAR/KY - allows to treat the black hole angular momentum as a parameter.)

For small distances to the black hole, light-bending effects must be important (Miniutti \& Fabian 2004) and the primary photons emerging from the flare source are directed down to the disk without reaching a very far distance from the flare. The light-bending is an effect of the curved space-time in the direct vicinity of the black hole, and becomes weaker for flares at larger disk radii. We also assume that the intrinsic radiation is collimated inside an irradiation cone limited by $\theta=60^{\circ}$. A possible interpretation of the irradiation cone can be given by assuming that the primary emission of the flare is not isotropic. A simple argument for such an assumption has been given by Nayakshin (2007) stating that magnetic flares occurring above the accretion disk of AGN are as likely to be anisotropic as solar flares. Nayakshin (2007) supports this argument with a simple consideration of the radiative transfer inside the coronal flare source. It is likely that at least for flares in the innermost region of the accretion disk both effects play a role.

The results we present in this paper show that detailed radiative transfer calculations for the reprocessed flare emission and its modifications due to the relativistic effects in the vicinity of the black hole are computationally feasible with current technology. They deliver a vast variety of results for the time-dependent spectral energy distributions and for the flare lightcurves. To apply them to a full extend to the observations of AGN, future generations of X-ray observatories are necessary. The models we presented here then allow to put important geometrical constraints on the flaring activity in the accretion flow of supermassive black holes.

Acknowledgements. This work was supported in part by the grant 1P03D00829 of the Polish State Committee for Scientific Research, by the Laboratoire Europeén Associé Astrophysique Pologne-France, and by the ESA-PECS 
project No. 98040. R.G. is grateful to the Centre of Theoretical Astrophysics and to the Hans-Böckler-Stiftung. M.D. and V.K. gratefully acknowledge support from the Czech Science Foundation grants 205/05/P525 and 205/07/0052.

\section{References}

Abramowicz, M. A., Bao, G., Lanza, A., \& Zhang, X.-H. 1991, A\&A, 245, 454 Ballantyne D. R., \& Ross R. R. 2002, MNRAS, 332, 777

Ballantyne D. R., Ross R. R., \& Fabian A. C. 2001, MNRAS, 327, 10

Blaes, O., Hirose, S., \& Krolik, J. H. 2007, ApJ, 664, 1057

Collin S., Coupé S., Dumont A.-M., Petrucci P.-O., \& Różańska A. 2003, A\&A, 400, 437

Czerny B., \& Goosmann R. 2004, A\&A, 428, 353

Czerny, B., Różańska, A., Dovčiak, M., Karas, V., \& Dumont, A.-M. 2004, A\&A, 420, 1

Dabrowski Y., \& Lasenby A. N. 2001, MNRAS, 321, 605

Dovčiak, M. 2004, Radiation of accretion disks in strong gravity, $\mathrm{Ph}$. D. Thesis, Charles University, Prague, Czech Republic

Dovčiak M., Karas V., \& Yaqoob T. 2004, ApJS, 153, 205

Dovčiak M., Karas V., Matt, G., Goosmann, R. W. 2007, A\&A, submitted

Dumont A.-M., Abrassart A., \& Collin S. 2000, A\&A, 357, 823

Dumont A.-M., Collin S., Paletou F., et al. 2003, A\&A, 407, 13

Fabian A. C., Vaughan S., Nandra K., et al. 2002, MNRAS, 335, L1

Galeev A. A., Rosner R., \& Vaiana G. S. 1979, ApJ, 229, 318

George, I. M., \& Fabian, A. C. 1991, MNRAS, 249, 352

Goosmann R. W., Czerny B., Mouchet M., et al. 2006a, A\&A, 454, 741

Goosmann R. W., Czerny B., Mouchet M., et al. 2006b, Astron. Nachr., 327, 977

Goosmann R. W., Czerny B., Karas V., \& Ponti G. 2007, A\&A, 466, 865

Guainazzi M., Bianchi S., \& Dovčiak M. 2006, Astron. Nachr., 327, 1032

Haardt, F., Maraschi, L., \& Ghisellini, G. 1994, ApJ, 432, L95

Henri, G., \& Pelletier, G. 1991, ApJ, 383, L7

Iwasawa K., Fabian A. C., Young A. J., Inoue H., \& Matsumoto C. 1999, MNRAS, 306, L19

Iwasawa K., Miniutti G., \& Fabian A. C. 2004, MNRAS, 355, 1073

Kaastra J. S., Steenbrugge K. C., Crenshaw D. M., et al. 2004, A\&A, 422, 97
Ko, Y.-K., \& Kallman, T. R. 1994, ApJ, 431, 273

Larsson J., Fabian A. C., Miniutti G., \& Ross R. R., 2007, MNRAS, 376, 348

Lubiński P., \& Zdziarski A. A. 2001, MNRAS, 323, L37

Martocchia A., \& Matt G. 1996, MNRAS, 282, L53

Martocchia A., Karas V., \& Matt G. 2000, MNRAS, 312, 817

Matt, G., Fabian, A. C., \& Ross, R. R. 1993a, MNRAS, 264, 839

Matt, G., Fabian, A. C., \& Ross, R. R. 1993b, MNRAS, 262, 17

Merloni, A., \& Fabian, A. C. 2001, MNRAS, 328, 958

Miller K. A., \& Stone J. M. 2000, ApJ, 534, 398

Miller L., Turner T. J., Reeves J. N., et al. 2006, A\&A, 453, L13

Miniutti G., \& Fabian A. C. 2004, MNRAS, 349, 1435

Miniutti G., Fabian A. C., Anabuki N., et al. 2007, PASJ, 59S, 315

Nayakshin, S. 2007, MNRAS, 376, L25

Nayakshin S., \& Kazanas D. 2002, ApJ, 567, 85

Nayakshin, S., Kazanas, D., \& Kallman, T. R. 2000, ApJ, 537, 833

Novikov I. D., \& Thorne K. S. 1973, in Black Holes, ed. C. DeWitt, \& B. DeWitt (New York: Gordon \& Breach)

Page D. N., \& Thorne K. S. 1974, ApJ, 191, 499

Ponti G., Cappi M., Dadina M., \& Malaguti G. 2004, A\&A, 417, 451

Raymond, J. C. 1993, ApJ, 412, 267

Różańska A., \& Czerny B. 1996, Acta Astron., 46, 233

Różańska A., Czerny B., Życki P. T., \& Pojmański G. 1999, MNRAS, 305, 481

Różańska, A., Dumont, A.-M., Czerny, B., \& Collin, S. 2002, MNRAS, 332, 799

Tanaka Y., Nandra K., Fabian A. C., et al. 1995, Nature, 375, 659

Tombesi F., De Marco B., Iwasawa K., et al. 2007, A\&A, 467, 1057

Turner, N. J. 2004, ApJ, 605, L45

Turner T. J., Miller L., George I. M., \& Reeves J. N. 2006, A\&A, 445, 59

Wilms J., Reynolds C. S., Begelman M. C., et al. 2001, MNRAS, 328, L27

Young A. J., Lee J. C., Fabian A. C., et al. 2005, ApJ, 631, 733

Zycki, P. T., \& Czerny, B. 1994, MNRAS, 266, 653

Życki P. T., \& Niedzwiecki A. 2006, in The Central Engine of Active Galactic Nuclei, ed. L. C. Ho, \& J.-M. Wang (San Francisco: ASP), in press [arXiv: astro-ph/0612597]

Zycki, P. T., Krolik, J. H., Zdziarski, A. A., \& Kallman, T. R. 1994, ApJ, 437, 597 